\title{
Commentary: Novel Delivery Approaches for Antiepileptic Drugs: Hope and Hurdles
}

\author{
Dieter Schmidt* and Gregory L. Holmes ${ }^{\dagger}$ \\ *Epilepsy Research Group, Berlin, Germany, ${ }^{\dagger}$ Dartmouth Medical School, Hanover, New Hampshire 03755
}

There is no compelling evidence that the ability of antiepileptic drugs (AEDs) to control seizures of newly treated epilepsy has improved dramatically since the introduction of carbamazepine in the 1970s. Although there have been advances in tolerability and safety with the introduction of modern AEDs, the modern drugs are similar to the older generation of drugs in efficacy against new-onset epilepsy. ${ }^{1,2}$ In addition, there is no convincing evidence that current AEDs are reducing the proportion of patients with drug-resistant epilepsy. ${ }^{3}$ Given that epilepsy is often (although not invariably) extensively drug resistant to several AEDs with different mechanisms of action, it may be worthwhile to consider other avenues than new mechanisms of drug action to improve seizure outcome. For example, more effective drug delivery to the epileptogenic brain tissue may improve seizure control. In this issue of Neurotherapeutics, several experts in the field review exciting advances in drug delivery that may eventually be beneficial for better delivery of AEDs currently under development for the treatment and prevention of epilepsy.

One of the biggest obstacles to drug delivery to the brain is the blood-brain barrier (BBB). It is remarkable that $98 \%$ of small molecule drugs and $100 \%$ of large molecule drugs do not cross the BBB. Developing strategies to circumvent the BBB are urgently needed. The three major developments of the brain drug delivery roadmap are 1) transcranial brain drug delivery, 2) BBB disruption, and 3) endogenous BBB transporters.

\section{TRANSCRANIAL BRAIN DRUG DELIVERY}

The only noninvasive delivery is through the transnasal route. The drug enters the olfactory CSF via absorption across the olfactory nasal epithelium. Water soluble drugs do not cross the olfactory epithelium, unless injured locally with a volume of more than $1000 \mu \mathrm{L}$ into the nostril. Other, more traumatic avenues include intracerebroventricular or intracerebral applications, which are clearly reserved-if ever-to life-threatening status epilepticus. In addition, convection-enhanced delivery (see below) may be used.

\section{BBB DISRUPTION}

The BBB can be disrupted through hyperosmolar, vasoactive substances or ultrasonic exposure. None of these procedures has been evaluated for AED delivery in humans. It would be of great interest to know if vasoactive drugs in clinical use (e.g., sildenafil) lend themselves to transient disruption of the BBB, for better delivery of AEDs. Recent research has compellingly highlighted the involvement of BBB permeability in the development of epilepsy. Disruption of the BBB has potential risks, however. Breakdown of the BBB can alter homeostatic environment of the brain and can pathologically alter neural activity, by allowing entrance of intravascular proteins (e.g., albumin), and altered electrolyte levels (e.g., increased potassium), into the extracellular space. ${ }^{4}$

\section{ENDOGENOUS BBB TRANSPORTERS}

A number of small and large molecules could serve as endogenous BBB transporters. Blood vessels of the brain, which form the BBB, have receptor-transport systems for endogenous peptides. Using a Trojan horse technology, drugs can be delivered to the brain by attaching them to a molecular Trojan horse, which enters the brain via the endogenous transport system of the BBB. In addition, genetic engineering of bifunctional recombinant fusion proteins would allow the efficient transfer of receptor-associated protein across the BBB.

\section{BRAIN GENE DELIVERY WITHOUT VIRUSES}

Trojan horse liposomes may supply promoter-driven organ-specific gene expression without using viruses in the adult rhesus monkey. Crossing the BBB with compounds with antiepileptic activity using gene delivery systems is a future goal.

\section{INTRAVENTRICULAR AND INTRACEREBRAL DELIVERY}

Dr. Barcia (pages 337-343) reviews strategies for intraventricular continuous infusion and intracerebral de- 
livery of AEDs in the kindling model. The major general concerns for intraventricular continuous infusion are, first, that is a very invasive procedure and, second, that it may work in small brains of rodents but is less likely to work in the large human brain. Intracerebral delivery of AEDs is invasive, and it may work only in circumscribed lesional epilepsy. The concern here is that partial epilepsy (despite its name) is often associated with widespread network changes of the brain that may be more difficult to reach with intracerebral delivery.

\section{CONVECTION-ENHANCED DELIVERY}

Dr. Rogawski (pages 344-351) reviews convectionenhanced delivery, in which a drug is pumped into the interstitial space of the brain under constant pressure. A major concern, in addition to those discussed by Dr. Rogawski, is the lack of clinical and experimental experience with AEDs.

\section{INTRANASAL DELIVERY OF TRH NANOPARTICLES}

Dr. Kubek (pages 359-371) shows that seizures induced by kindling in the rat can be attenuated through intranasal delivery of nanoparticles of the neuropeptide thyrotropin releasing hormone (protirelin). Problems in neuropeptide development, apart from getting them across the BBB, include rapid metabolism, lack of sustained site-specific bioavailability, adverse effects, and cost and quality control issues in producing neuropeptides.

\section{INTRANASAL DELIVERY OF AEDS}

Dr. Wermeling (pages 352-358) reviews intranasal delivery of AEDs. In general, nasal delivery has a number of advantages, including easy administration, rapid systemic uptake, and prompt and safe pharmacological action. For epilepsy, however, clinical experience for nasal drug treatment is limited to benzodiazepines. Dr. Wermeling notes that the evidence for benzodiazepines is limited largely to midazolam, and one study with diazepam, with no published evidence for clonazepam and lorazepam. It remains unclear which benzodiazepines are best suited for nasal use. Well designed clinical studies are needed to evaluate the clinical role of nasal use of benzodiazepines.

\section{SYSTEMIC DELIVERY OF GALANIN-BASED NEUROPEPTIDES}

Dr. White (pages 372-380) reviews the use and delivery of galanin, neuropeptide Y, somatostatin, and other neuropeptides for epilepsy. Galanin, a large and metabolically unstable neuropeptide, does not cross the BBB.
Neither cationization nor lipidization produces galanin analogs that are potent following systemic delivery. However, a patented chemistry approach allows the production of CNS-active galanin analogs, including NAX 5055, which has been shown to protect against subcutaneous pentylenetetrazole-induced clonic seizure, but not maximal electroshock-induced tonic extension. Dr. White concludes that the technology has the potential to create neuropeptide-based therapies for epilepsy and possibly for other CNS disorders.

\section{CONCLUSION}

In summary, many of the most promising preclinical data on novel approaches to improve antiepileptic drug delivery are aimed at either disrupting or bypassing the BBB, including Trojan horse technology that would allow targeting of large (and water insoluble) molecules through the BBB to epileptic brain regions responsible for seizure generation. The most important aspect is that such novel delivery approaches will allow clinical testing for antiepileptic activity of large molecules that were previously barred from development of epilepsy drugs. There may be a whole new world of effective epilepsy drugs that were excluded from experimental and clinical evaluation simply because they could not cross the BBB. Researchers and practitioners in the field eagerly await the results—-with cautious optimism and with high hopes.

Ultimately, our best chance for effectively treating and preventing epilepsy will involve therapies that, on the one hand, safely and specifically regulate seizure generation and, on the other hand, enhance the safety and tolerability by avoiding systemic toxicity. Once clinical trials of new delivery techniques have been conducted, the hope is that we will soon have novel epilepsy therapeutics that successfully and safely stop seizures in more patients than is possible today.

Acknowledgments: Supported in part by grants from the National Institutes of Health, National Institute of Neurological Disorders and Stroke (NS041595 and NS044296), the Epilepsy Foundation, and Citizens United for Research in Epilepsy (CURE).

\section{REFERENCES}

1. Marson AG, Appleton R, Baker GA, et al. A randomised controlled trial examining the longer-term outcomes of standard versus new antiepileptic drugs. The SANAD trial. Health Technol Assess 2007; 11:iii-X, 1 .

2. Tudur Smith C, Marson AG, Chadwick DW, Williamson PR. Multiple treatment comparisons in epilepsy monotherapy trials. Trials 2007;8:34.

3. Schmidt D, Löscher W. Drug resistance in epilepsy: putative neurobiologic and clinical mechanisms. Epilepsia 2005;46:858-877.

4. Seiffert E, Dreier JP, Ivens S, et al. Lasting blood-brain barrier disruption induces epileptic focus in the rat somatosensory cortex. J Neurosci 2004;24:7829-7836. 